\title{
Association of early PSA decline and time to PSA progression in abiraterone acetate- treated metastatic castration-resistant prostate cancer; a post-hoc analysis of Japanese phase 2 trials
}

\author{
Masahiko Nakayama ${ }^{1 *}$, Hisanori Kobayashi ${ }^{1}$, Tomihiro Takahara ${ }^{1}$, Ryo Oyama ${ }^{2}$, Keiichiro Imanaka²
} and Kazutake Yoshizawa'

\begin{abstract}
Background: Previous studies have demonstrated an association between prostate-specific antigen (PSA) kinetics and predictive value for treatment outcomes. Abiraterone acetate (AA) is a newly approved cytochrome-P450C17 inhibitor for treatment of metastatic castration-resistant prostate cancer (MCRPC), and few studies have evaluated PSA kinetics using AA so far. Results of a study evaluating PSA kinetics in the beginning of AA and enzalutamide responded chemotherapy-treated patients suggested different trends between the drugs. PSA kinetics of AA-treated patients has been reported using large datasets; however, no studies which have fully evaluated PSA kinetics in the beginning treatment. The present study aimed to assess the PSA kinetics and relationship between the PSA kinetics and PSA progression in chemotherapy-naïve and chemotherapy-treated mCRPC patients receiving AA.

Methods: We used two Japanese phase II trial datasets: JPN-201, chemotherapy-naïve mCRPC $(n=48)$ and JPN-202, chemotherapy-treated mCRPC $(n=46)$. PSA kinetic parameters were calculated using actual PSA values measured every 4 weeks, and a subgroup analysis was performed to evaluate the influence of early PSA response on time to PSA progression (TTPP). In addition, we used a Cox proportional hazard model to investigate the influence of variables on TTPP.

Results: PSA declined from week 4 but took more time to achieve nadir. PSA kinetic parameters were different between the datasets, mean time to PSA nadir was $5.3 \pm 5.6$ and $2.0 \pm 3.4$ months, and TTPP was $9.5 \pm 7.4$ and $3.8 \pm 4.8$ months in JPN-201 and JPN-202, respectively. In the subgroup analysis of week 4 PSA decline status, Kaplan-Meier curves for TTPP were similar between early responders and non-progression patients in JPN-201 (median, 9.2 vs. 6.5 months, respectively) but separated in JPN-202 (median, 3.7 vs. 1.9 months, respectively). According to univariate Cox regression analysis, achievement of PSA response ( $\geq 50 \%$ ) at week 12 was associated with TTPP in the both trials, but the hazard ratio of PSA decline ( $\geq 30 \%)$ at week 4 was not significant in JPN-201.
\end{abstract}

Conclusions: Our results suggest that PSA kinetics were not comparable and early PSA response showed different association to TTPP according to prior history of chemotherapy.

Trial registration: The original trials are registered at ClinicalTrials.gov. The identifiers are; JNJ-212082-JPN-201, registered 20 December 2012 and JNJ-212082-JPN-202, registered 30January 2013.

Keywords: Abiraterone acetate, Castration-resistant prostate cancer, Kinetics, Prostate-specific antigen, Cox proportional hazard model

\footnotetext{
* Correspondence: mnakayam@its.jnj.com

${ }^{1}$ Medical Affairs Division, Janssen Pharmaceutical K.K., 5-2, Nishi-kanda,

3-Chome, Chiyoda-ku, Tokyo 101-0065, Japan

Full list of author information is available at the end of the article
} 


\section{Background}

Globally, the estimated incidence of prostate cancer was approximately 1.4 million in 2013. There was a 3-fold increase in this incidence from 1990 to 2013, together with aging and population growth [1]. Since Huggins et al. discovered that prostate cancer growth is stimulated by androgens, castration has been the mainstay of advanced-stage prostate cancer treatment [2]; however, most patients develop resistance to castration.

Abiraterone acetate (AA) is a prodrug of abiraterone, which is a first-in-class inhibitor of cytochromeP450C17 that plays a role in the mechanism of castration resistance by de novo androgen synthesis [3]. It is approved with prednisone for treatment of metastatic castration-resistant prostate cancer (mCRPC) worldwide. AA plus prednisone significantly prolonged overall survival (OS) compared with placebo plus prednisone for treatment of chemotherapy-naïve and chemotherapy-treated mCRPC in pivotal global trials $[4,5]$. In Japan, two singlearm, open-label, phase II trials were separately conducted for the purpose of obtaining local registration [6, 7].

Prostate-specific antigen (PSA) is a reliable, sensitive, and easy to measure biomarker for prostate cancer and is therefore widely used for evaluation of treatment in practice $[8,9]$. PSA kinetics has been studied in androgen deprivation therapy using anti-androgens or taxanes to analyze its predictive value for time-dependent outcomes such as OS and disease progression. Several studies have reported strength of PSA decline and its predictive value for OS, although certain results were controversial [9-11]. Recently, Caffo et al. reported the PSA kinetics of AA and enzalutamide responders and demonstrated different trends with regard to PSA kinetics between the drugs in chemotherapy-treated mCRPC patients [12]. However, patient number was limited, and PSA kinetics of chemotherapy-naïve mCRPC patients was not reported. Xu et al. also reported PSA kinetics of AAtreated mCRPC patients separately by chemotherapynaïve and -treated populations. However, PSA kinetics within 12 weeks was not evaluated because the original trials measured PSA values every 12 or 16 weeks [13]. Thus, PSA kinetics in AA-treated mCRPC patients has not been fully clarified so far.

Moreover, the Prostate Cancer Clinical Trial Working Group (PCWG2) advises to ignore early PSA changes to avoid detecting continuing rise of PSA level and increasing in size before it regress [8]. However, some researchers reported early PSA decline, and its predictive value was possibly different by patient backgrounds and treatment $[12,14,15]$. In addition, the clinical practice in Japan, most of mCRPC patients are primary followed by monthly PSA testing, so, there is a potential of over use of early response as predictive factor for efficacy regardless the PCWG2 criteria.
The aims of the present study were to assess the PSA kinetic profile, and the relationship between PSA kinetics calculated based on actual PSA values measured 4 weeks and 12 weeks and PSA progression in chemotherapy-naïve and chemotherapy-treated mCRPC patients receiving AA.

\section{Methods}

Data source

This post-hoc study was conducted using datasets from two Japanese phase II trials of AA for mCRPC: JPN-201 (ClinicalTrials.gov Identifier, JNJ-212082-JPN-201) that included chemotherapy-naïve patients $(n=48)$ and JPN202 (ClinicalTrials.gov Identifier, JNJ-212082-JPN-202) that included patients who received docetaxel-based chemotherapy $(n=46)$. Results from the original trials are available elsewhere $[6,7]$. Major inclusion criteria were as follows: men with $\mathrm{mCRPC}$ aged $\geq 20$ years with a PSA level of $\geq 5 \mathrm{ng} / \mathrm{mL}$ and an Eastern Cooperative Oncology Group Performance Status (ECOG-PS) score of 0 or 1 for JPN-201 and 0 to 2 for JPN-202, histologically or cytologically confirmed adenocarcinoma of the prostate without neuroendocrine differentiation or small cell histology, and testosterone levels of $\leq 50 \mathrm{ng} / \mathrm{dL}$ by medical or surgical castration. Eligible patients were orally administered AA $1000 \mathrm{mg}$ with 10-mg prednisolone per day. For patients with medical castration, castration was maintained by using a luteinizing hormonereleasing hormone (LHRH) agonist throughout the study. PSA was assessed at baseline and every 28 days after the AA dose.

The primary endpoint of the original trials was the proportion of patients achieving a PSA decline of $\geq 50 \%$ from baseline within 12 weeks of therapy in accordance with the Prostate-Specific Antigen Working Group criteria (PSAWG) [16].

An independent ethics committee or institutional review board approved the protocols of original trials and informed consent forms, and the trials were conducted in accordance with the ethical principles in the Declaration of Helsinki and consistent with the Good Clinical Practices and applicable regulatory requirements. All patients or their legally acceptable representatives provided written informed consent, which included consent for post-hoc analysis before entering the original trials.

\section{PSA values used in the study and definitions of PSA} kinetic parameters

We used PSA values obtained after each 28-day cycle from baseline (day 0) to end of treatment (EOT) or study cutoff date (the longest duration was 785 days) to calculate the PSA kinetic parameters.

The following PSA kinetic parameters were calculated, as defined in the present study: 
(1) Maximum percentage PSA decline (\%; defined as nadir PSA value/baseline PSA value $\times 100)$

(2) Time to PSA nadir (months; defined as duration from baseline to PSA nadir)

(3) Nadir PSA value (ng/mL; defined as the minimum PSA value during the treatment period)

(4)EOT PSA value (ng/mL; defined as the last PSA value at EOT)

(5)PSA response ( $\geq 50 \%$ ) according to PCWG2 criteria

(6)PSA response ( $\geq 30,50$, and $90 \%)$ at week 12 according to PCWG2 criteria

(7) Time to PSA progression (TTPP; months, defined as duration from baseline to the day of PSA progression according to PCWG2 criteria)

The purpose of the original studies was to demonstrate similarity with global trials, response criteria needed to be identical with global trials used. In the present study, we referred PCWG2 criteria instead of PSAWG criteria, because it no longer used in the current clinical practice.

\section{Statistical analysis}

Findings from JPN-201 and JPN-202 were separately analyzed. Patient demographics, baseline characteristics, and PSA kinetic parameters were descriptively summarized using mean, standard deviation (SD), and percentage values. The percentage PSA change was longitudinally described based on mean (SD) and $95 \%$ confidence intervals (CI) at each time point to characterize the post-treatment PSA kinetics.

We analyzed the impact of early PSA decline on TTPP. Because treatment was repeated every 28 days, and PSA was measured on the first day of each cycle in JPN-201 and JPN-202, the PSA value at week 4 was the earliest PSA value available to assess PSA transition. We subdivided the percent PSA change at week 4 into the following three subgroups: (1) PSA decline $\leq-30 \%$ (30\%-decline), (2) PSA $>-30$ and $<25 \%$ (non-decline), and (3) PSA elevation $\geq 25 \%$ (25 \%-elevation). Furthermore, TTPP was analyzed based on PSA response ( $\geq 50 \%$ ) at week 12 according to the PCWG2 criteria. In addition, impact of the PSA kinetic parameters on TTPP was investigated using a Cox proportional hazard model to obtain the hazard ratio (HR) and its $95 \%$ CI. TTPP curves were developed using Kaplan-Meier method.

All statistical analyses were performed using $R$ version 3.1.0 (a language and environment for statistical computing; R Foundation for Statistical Computing, Vienna, Austria).

\section{Results}

Baseline demographics and characteristics

In the present study, data from a total of 94 patients were analyzed: 48 patients from JPN-201 and 46 from
JPN-202. Baseline demographics and characteristics are summarized in Table 1. In JPN-201, the median age was 70 years, 83.3 of the patients had ECOG-PS scores of 0 , and $89.6 \%$ had a Gleason score of $\geq 8$. In JPN-202, the median age was 71 years, 52.2, 34.8, and $13.0 \%$ of the patients had ECOG-PS scores of 0, 1, and 2, respectively, and $78.3 \%$ had a Gleason score of $\geq 8$.

In JPN-202, all patients had received docetaxel-based chemotherapy at study entry.

\section{Abiraterone compliance, dose reducations and interaptions}

In JPN-201, $91.7 \%(44 / 48)$ had > $90 \%$ compliance with AA. Dose reductions were required for 3 (6.3\%) patients. Dose interruptions were required for 21 (45.7\%) patients, once for 13 and 9 patients required 2 or more dose interruptions.

In JPN-202, $93.5 \%(43 / 46)$ had $>90 \%$ compliance with AA. Dose reductions were required for 6 (13.0\%) patients. Dose interruptions were required for 22 (47.8\%) patients, 8 patients required 2 or more dose interruptions.

No patients discontinued study treatment because of poor compliance in the both trials.

\section{PSA kinetic parameter values}

PSA values were well followed up, PSA progression was confirmed $75(36 / 48)$ and $10 \%(5 / 48)$ was censored within the first year in JPN-201. In JPN-202, PSA progression was confirmed $93(43 / 46)$ and $4 \%(2 / 46)$ was censored within the first year.

In JPN-201, PSA rapidly decreased from week 4 onwards, and the mean PSA decline from baseline value was $51.6 \%$ (SD: 41.2) (Fig. 1a). Figure 1b depicts the percentage change of PSA transition in each patient. The PSA kinetic parameters are shown in Table 2. At week 4, 36 patients achieved a $30 \%$-decline, 9 patients showed a non-decline, and 3 patients showed a $25 \%$-elevation. PSA responses ( $\geq 50 \%$ ) were confirmed in 28 of 36 patients who achieved 30-decline, 2 patients who showed a non-decline, and none of the $25 \%$-elevation patients. Figure $2 \mathrm{a}$ shows the TTPP according to the week 4 percent PSA change subgroups. The median TTPP was 9.2 months for $30 \%$-decline, 6.5 months for non-decline, and 1.0 month for $25 \%$-elevation. In addition, of the 9 non-decline patients, 1 continued to have a PSA response and 6 showed PSA progression at EOT. Other 2 patients were censored by discontinuing of the treatment. Figure $2 \mathrm{~b}$ shows TTPP according to the PSA response $(\geq 50 \%)$ at week 12 . The median TTPP was 11.1 months for responders, 1.9 month for patients with PSA progression, and 6.5 months for others (nonresponse and non-progression). 
Table 1 Baseline patient demographics and characteristics

\begin{tabular}{|c|c|c|}
\hline & $\begin{array}{l}\text { Chemotherapy-naive } \\
\text { JPN-201 }(n=48)\end{array}$ & $\begin{array}{l}\text { Chemotherapy-treated } \\
\text { JPN-202 }(n=46)\end{array}$ \\
\hline Median age (range), years & $70(46-89)$ & $71(51-83)$ \\
\hline \multicolumn{3}{|l|}{ Gleason score } \\
\hline$<7$ & $0 \%$ & $0 \%$ \\
\hline 7 & $8.3 \%$ & $17.4 \%$ \\
\hline$\geq 8$ & $89.6 \%$ & $78.3 \%$ \\
\hline Unknown & $2.1 \%$ & $4.3 \%$ \\
\hline \multicolumn{3}{|l|}{ ECOG-PS score } \\
\hline 0 & $83.3 \%$ & $52.2 \%$ \\
\hline 1 & $16.7 \%$ & $34.8 \%$ \\
\hline 2 & - & $13.0 \%$ \\
\hline \multicolumn{3}{|l|}{ Extent of disease } \\
\hline Bone & $91.7 \%$ & $95.7 \%$ \\
\hline Hepatic & $2.1 \%$ & $4.3 \%$ \\
\hline Lymphatic & $39.6 \%$ & $37.0 \%$ \\
\hline Pulmonary & $0 \%$ & $10.9 \%$ \\
\hline Other & $0 \%$ & $6.5 \%$ \\
\hline Median months from initiating LHRH agonist to first dose (range) & $21.91(6.2-191.6)$ & $41.23(4.4-182.8)$ \\
\hline Median baseline PSA (range), ng/mL & $31.40(6.0-469.0)$ & $147.00(7.2-1450.0)$ \\
\hline Median baseline hemoglobin (range), g/dL & $12.85(10.2-15.2)$ & $11.80(9.0-14.9)$ \\
\hline Median LDH (range), IU/L & $212.0(164-1045)$ & $211.0(122-723)$ \\
\hline Median alkaline phosphatase (range), IU/L & $292.0(139-2643)$ & $327.0(69-2991)$ \\
\hline \multicolumn{3}{|l|}{ No. of previous chemotherapy regimens } \\
\hline 1 & - & $39.1 \%$ \\
\hline 2 & - & $60.9 \%$ \\
\hline
\end{tabular}

In JPN-202, the mean PSA decline at week 4 was $14.9 \%$ (SD: 59.1) (Fig. 1c). Figure 1d depicts the percentage change of PSA transition for each patient. The PSA kinetic parameters are shown in Table 2. At week 4, 21 patients achieved a $30 \%$-decline, 14 patients showed a non-decline and 11 showed a $25 \%$-elevation. PSA responses ( $\geq 50 \%)$ were confirmed in 13 of $2130 \%$-decline patients, while none of the patients had PSA responses in the other subgroups. Figure 3a shows the TTPP according to the subgroups; the median TTPP was 3.7 months for $30 \%$-decline, 1.9 months for non-decline, and 1.0 month for $25 \%$-elevation. All non$30 \%$-decline patients had PSA progression at EOT. Figure 3b shows the TTPP according to PSA response $(\geq 50 \%)$ at week 12. The median TTPP was 4.6 months for responders, 1.9 months for patients with PSA progression, and 4.6 months for others (non-response and non-progression).

\section{Results of univariate Cox analyses}

Table 3 shows the results of univariate Cox regression analyses for TTPP. In JPN-201, all variables showed significance except PSA decline $(\geq 30 \%)$ at week 4 . In JPN-202, all variables showed significance except nadir PSA value and PSA response ( $\geq 90 \%)$ at week 12 .

\section{Discussion}

In this post-hoc analysis, we first assessed the PSA kinetic profile in AA plus prednisolone-treated mCRPC patients. There was a clear difference in the PSA kinetic profile between chemotherapy-naïve and chemotherapytreated patients. The majority of chemotherapy-naïve patients showed a rapid PSA decline at week 4, and additional 4 months were needed to reach PSA nadir. On the other hand, the majority of chemotherapytreated patients showed non-decline or $25 \%$ elevation at week 4 , and the mean time to reach PSA nadir was 2.0 months. Moreover, the percent change in the PSA values in chemotherapy-treated patients tended to be larger, and most of the patients experienced PSA progression within a year after treatment initiation (Fig. 1). The mean TTPP was approximately 4 months, which was 

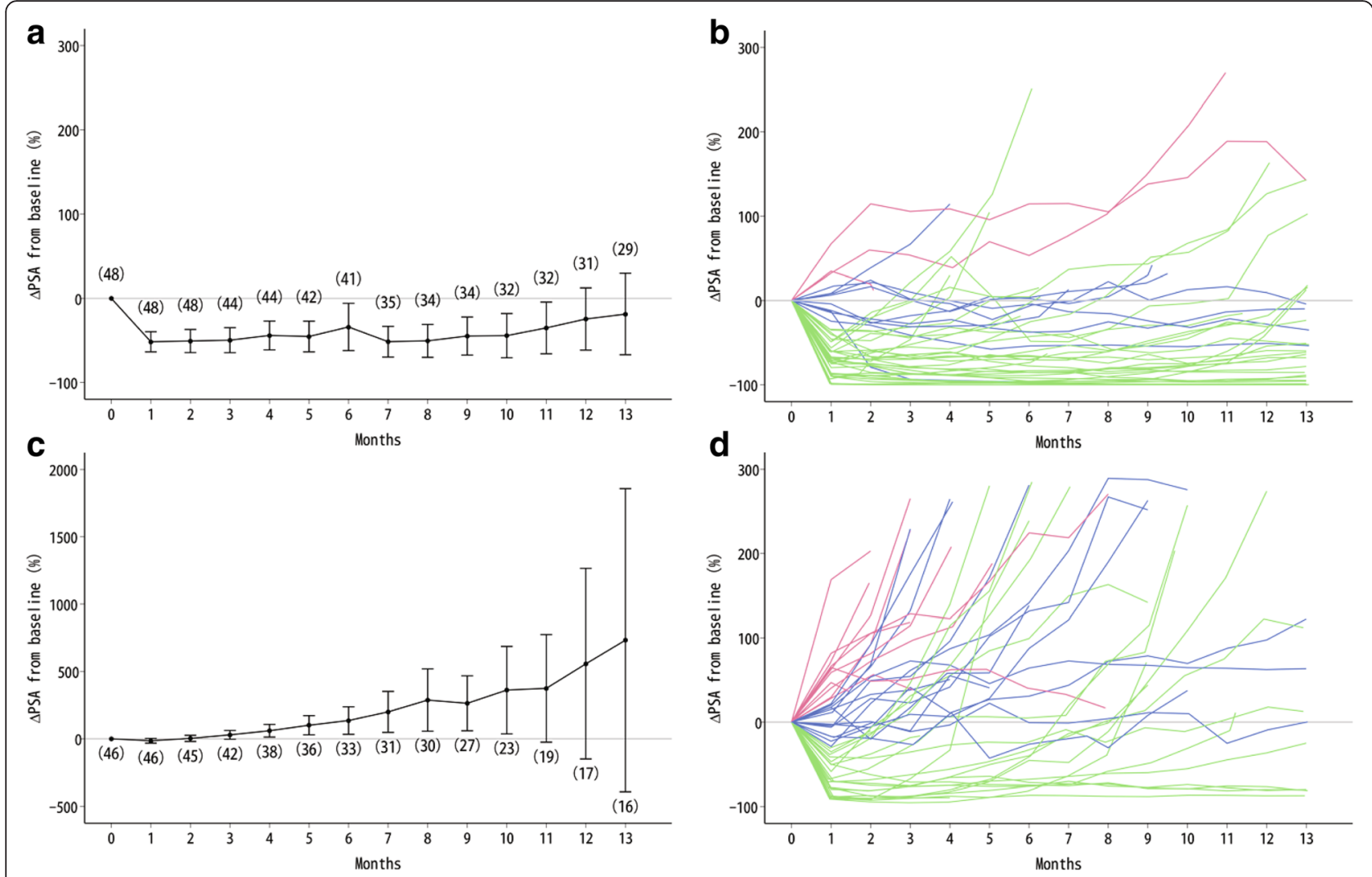

Fig. 1 Percent PSA transition from baseline to month 13. a Mean percent PSA change in JPN-201, (n). b Percent PSA change for each patient in JPN-201; green, PSA $\leq-30 \%$ (30\%-decline); blue, -30\%<PSA < $25 \%$ (non-decline); red, PSA $\geq 25 \%$ (25\%-elevation) according to PSA change at week 4. c Mean percent PSA change in JPN-202, (n). d Percent PSA change for each patient in JPN-202; green, PSA $\leq-30 \%$ (30\%-decline); blue, $-30 \%<$ PSA < $25 \%$ (non-decline); red, $25 \% \geq$ PSA (25 \%-elevation) according to PSA change at week 4 . Data are expressed as mean $\pm 95 \%$ Cl. Note: PSA changes of $\geq 300 \%$ and those after month 13 are not shown

approximately half of the value reported in JPN-201 (9.5 months). Differences in characteristics of patient populations with mCRPC may impact the PSA kinetic profile, and such PSA kinetic differences could mainly be associated with previous history of chemotherapy. The mechanisms related to the differences in PSA kinetic profiles between chemotherapy-naïve and chemotherapy-treated patients are unclear; however, it is recommended that PSA kinetics would be separately evaluated according to prior history of chemotherapy. Caffo et al. reported that the time to PSA nadir in responders among chemotherapy-treated mCRPC patients was 15.5 weeks with AA but 7.0 weeks with enzalutamide [12]. Moreover, the anti-tumor effects of both drugs are

Table 2 PSA kinetic parameters, mean \pm SD $(95 \%$ Cl)

\begin{tabular}{lll}
\hline & $\begin{array}{l}\text { JPN-201 }(n=48) \\
\text { Chemotherapy-naive }\end{array}$ & $\begin{array}{l}\text { JPN-202 (n=46) } \\
\text { Chemotherapy-treated }\end{array}$ \\
\hline Maximum \% PSA decline & $64.4 \pm 38.3(53.3-75.5)$ & $19.7 \pm 59.4(2.0-37.3)$ \\
Time to PSA nadir (months) & $5.3 \pm 5.6(3.7-6.9)$ & $2.0 \pm 3.4(1.0-3.0)$ \\
Nadir PSA value $(\mathrm{ng} / \mathrm{mL})$ & $19.5 \pm 28.3(11.3-27.7)$ & $184.9 \pm 282.3(101.0-268.7)$ \\
PSA response $(\geq 30 \%)$ at week 12, $\mathrm{n}(\% ; 95 \% \mathrm{Cl})$ & $35(72.9 ; 58.2-84.7)$ & $15(32.6 ; 19.5-48.0)$ \\
PSA response $(\geq 50 \%)$ at week 12, $\mathrm{n}(\% ; 95 \% \mathrm{Cl})$ & $29(60.4 ; 45.3-74.2)$ & $13(28.3 ; 16.0-43.5)$ \\
PSA response $(\geq 90 \%)$ at week 12, $\mathrm{n}(\% ; 95 \% \mathrm{Cl})$ & $9(18.8 ; 8.9-32.6)$ & $2(4.3 ; 0.5-14.8)$ \\
PSA response $(\geq 50 \%)$ in treatment period, $\mathrm{n}(\% ; 95 \% \mathrm{Cl})$ & $30(62.5 ; 47.48-76.0)$ & $13(28.3 ; 16.0-43.5)$ \\
Time to PSA progression (months) & $9.5 \pm 7.4(7.4-11.6)$ & $3.8 \pm 4.8(2.4-5.2)$
\end{tabular}



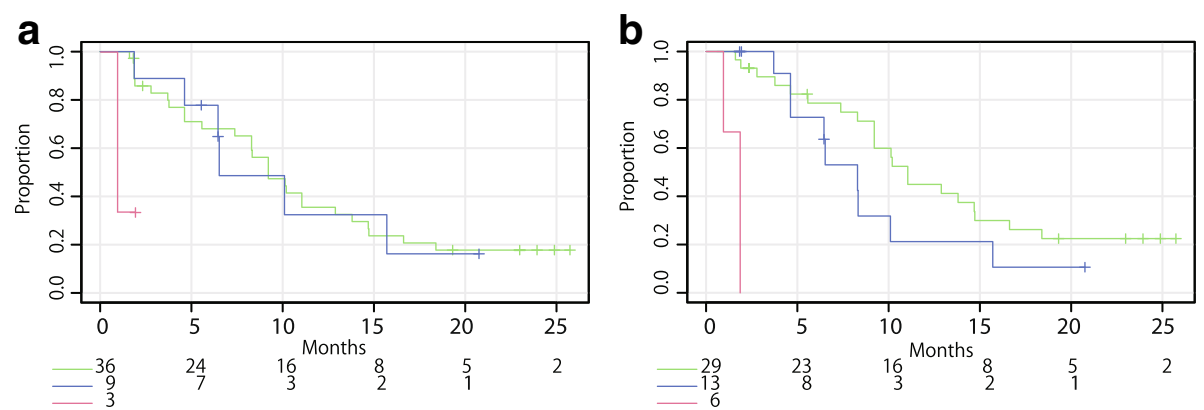

Fig. 2 Kaplan-Meier curves for time to PSA progression in JPN-201. a green, PSA $\leq-30 \%$ (30\%-decline); blue, $-30 \%<$ PSA < $25 \%$ (non-decline); red, PSA $\geq 25 \%$ (25 \%-elevation) according to PSA change at week 4. b green, PSA response ( $\geq 50$ \%); blue, PSA non-response/progression; red, PSA progression according to PSA response at week 12

derived from different mechanisms of action; abiraterone inhibits androgen synthesis at non-gonadal sites (adrenal gland and intratumorally) to reduce androgens to below castrate concentrations, while enzalutamide, an antiandrogen, directly binds androgen receptors to inhibit androgen receptor nuclear translocation [17]. These differences in the mechanisms of action might affect PSA kinetics.

In the present study, some patients showed a continuous stable level of PSA (Fig. 1b). In JPN-201, of the 9 (19\%) patients categorized as non-decline, most did not show large PSA elevation within 12 months. The TTPP curves were similar between non-decline and $30 \%$-decline patients (Fig. 2a). Thus, for chemotherapy-naïve patients without PSA progression at week 4, there would be a clinically similar chance of benefit from AA treatment. This is also supported by the result of Cox regression analysis, HR was significant for PSA response $(\geq 50 \%)$ at week 12 but not for PSA decline $(\geq 30 \%)$ at week 4 (Table 3).

On the other hand, in JPN-202, the non-decline TTPP curve was shorter than that of $30 \%$-decline and differed from that of $25 \%$-elevation (Fig. 3a). A half of the nondecline patients had rapid PSA progression; however, another half of the patients showed continuous stable levels of PSA (Fig. 1d), suggesting that patients with non-decline still had some potential of obtaining a clinical benefit from the treatment. The TTPP curves based on PSA response at week 12 were close between responders and patients with non-progression (Fig. 3b). Overall, further observation is recommended for patients in both population regardless obtaining PSA elevation at week 4.

In addition, temporal PSA elevation was recently reported with docetaxel treatment [18], which led to the phenomenon being referred to as "PSA flare," indicating a PSA elevation with initiation of an LHRH agonist. PSA flares were also reported with AA treatment $[19,20]$. According to the definition by Olbert et al., wherein a PSA flare is defined as initially rising PSA under therapy, dropping thereafter to values below baseline, 3 of 9 nondecline patients (Fig. 1b; blue lines) in JPN-201and 1 of 21 in JPN-202 (Fig. 1d; blue lines) were considered to have a PSA flare. PSA transition in such patients showed slow PSA elevations for 2 months, which declined below baseline levels within 1 to 2 months after the peak level. In taxane-based chemotherapy, the pattern of flare-up was slightly different. In the chemotherapy-
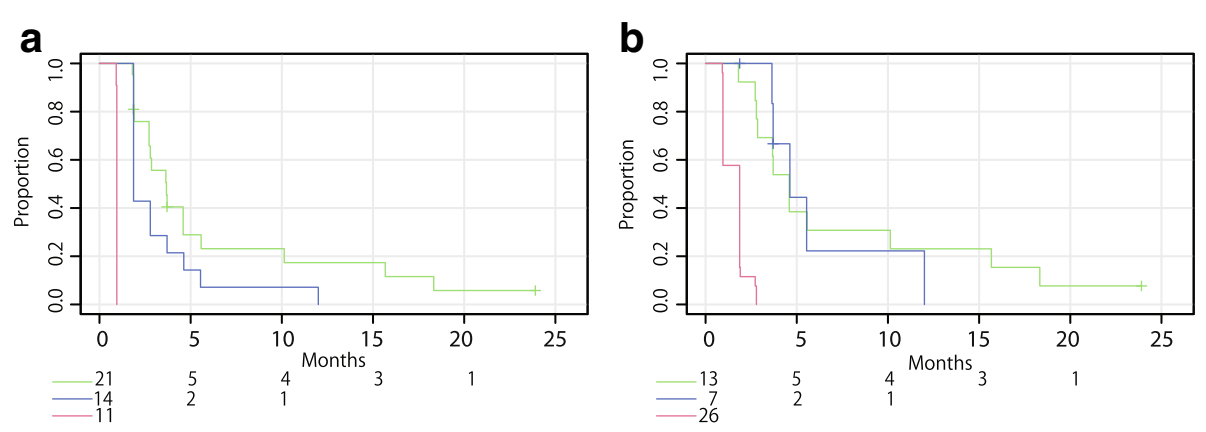

Fig. 3 Kaplan-Meier curves for time to PSA progression in JPN-202. a green, PSA $\leq-30 \%$ (30\%-PSA-decline); blue, $-30 \%<$ PSA $<25 \%$ (non-decline); red, PSA $\geq 25 \%$ (25\%-elevation) according to PSA change at week 4. b green, PSA response ( $\geq 50 \%$ ); blue, PSA non-response/progression; red, PSA progression according to PSA response at week 12 
Table 3 Results of univariate Cox analysis for time to PSA progression

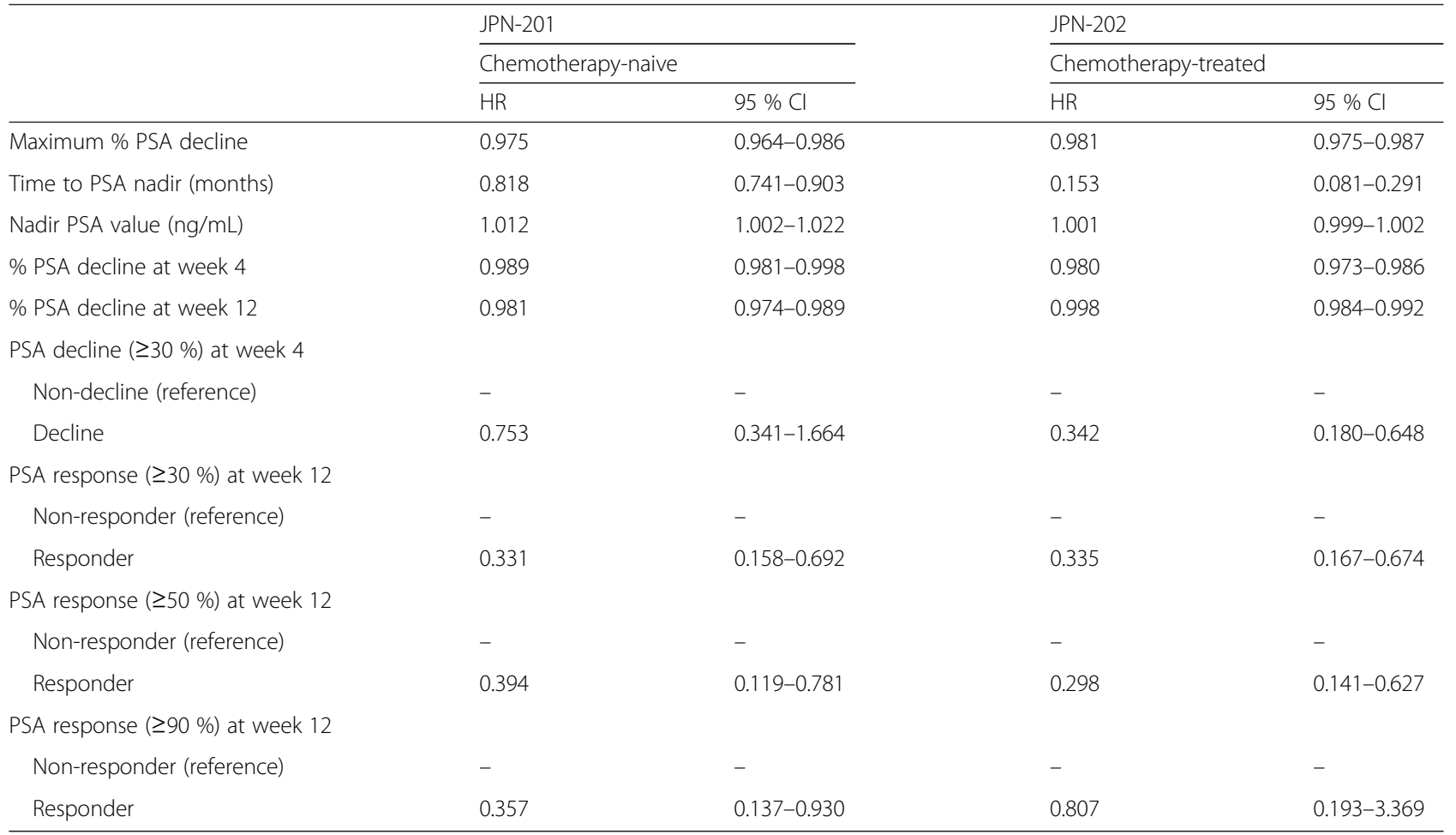

$\mathrm{Cl}$ confidence interval, $H R$ hazard ratio, PSA prostate-specific antigen

related PSA flare, a peak PSA level was reached within 1 month after the first dose, and peak PSA levels were detected 1 to 2 months earlier than in the present study [18, 21, 22].

In general, elevated or stable PSA levels are a key decision factor for treatment change in routine clinical practice. Based on the aforementioned results, careful consideration is recommended for evaluation of the efficacy and decision for treatment change in AA treatment.

Some limitations of the present study are as follows: TTPP is a well-known surrogate endpoint for OS and is easy to follow-up in clinical practice [8]. In the present study, investigation of OS was inappropriate because patient number and number of events were limited. Therefore, we alternatively evaluated TTPP. Nonetheless, the primary endpoint in both JPN-201 and JPN202 was PSA response rate at week 12; however, patient number was insufficient to apply statistical testing to analyze TTPP between the subgroups. Especially, patient number of non-30\%-decline was limited (11 in chemotherapy-naïve and 25 in chemotherapy-treated population). Moreover, in clinical practice, a wide variety of prior therapies, patient conditions, and concomitant use of drugs may have a potential impact on PSA kinetic profiles; it will therefore be important to reconfirm these results in clinical practice.

\section{Conclusions}

Our results suggest that PSA kinetics and impact of early PSA decline on TTPP were not comparable and early PSA response showed slightly different association to TTPP according to prior history of chemotherapy. However, this is simply a descriptive study for which practice changing assessment of response or predictor of failure just cannot be made, further studies are warranted to confirm these results.

\section{Abbreviations}

AA, abiraterone acetate; Cl, confidence interval; ECOG-PS, Eastern Cooperative Oncology Group Performance Status; EOT, end of treatment; HR, hazard ratio; $\mathrm{LHRH}$, luteinizing hormone-releasing hormone; $\mathrm{MCRPC}$, metastatic castrationresistant prostate cancer; OS, overall survival; PCWG2, Prostate Cancer Clinical Trials Working Group; PSA, prostate-specific antigen; PSAWG, ProstateSpecific Antigen Working Group; SD, standard deviation; TTPP, time to prostate-specific antigen progression

\section{Acknowledgements}

We would like to thank all the patients and their families and investigators who participated in JPN-201 and JPN-202. The names of the independent ethics committee or institutional review board approved the original trials were: Hokkaido University Hospital IRB, Gunma University Hospital IRB, Kumagaya General Hospital IRB, Asahi General Hospital IRB, Toho University Sakura Medical Center IRB, The Institutional Review Board of National Cancer Center, Kyorin University Hospital IRB, Kitasato University Hospital IRB, Yokosuka Kyosai Hospital IRB, Yokohama City University Hospital IRB, Niigata University Medical \& Dental Hospital IRB, Ishikawa Prefectural Central Hospital IRB, Osaka City University Hospital IRB, Kurashiki Central Hospital IRB, Kagawa University Hospital IRB, National Shikoku Cancer Center IRB, Kyushu University Hospital IRB, Osaka 
Medical Center for Cancer and Cardiovascular Diseases IRB, Kinki University Hospital IRB, Hara Sansin Hospital IRB and Gifu University Hospital IRB for JPN-201. For JPN-202, Hokkaido University Hospital IRB, Gunma University Hospital IRB, Kumagaya General Hospital IRB, Asahi General Hospital IRB, Toho University Sakura Medical Center IRB, Tokyo Women's University Hospital IRB, Kyorin University Hospital IRB, Kitasato University Hospital IRB, Yokosuka Kyosai Hospital IRB, Yokohama City University Hospital IRB, Niigata University Medical \& Dental Hospital IRB, Ishikawa Prefectural Central Hospital IRB, Osaka City University Hospital IRB, Kurashiki Central Hospital IRB, Kagawa University Hospital IRB, National Shikoku Cancer Center IRB, Kyushu University Hospital IRB and Kinki University Hospital IRB.

\section{Funding}

The original trials (JNJ-212082-JPN-201 and JNJ-212082-JPN-202) were conducted and funded by Janssen Pharmaceutical K.K.

\section{Availability of data and materials}

Janssen pharmaceutical, a pharmaceutical company of Johnson and Johnson, has a rights to the raw data of JNJ-212082-JPN-201 and JNJ212082-JPN-202. As part of our commitment to the implementation of the joint EFPIA/PhRMA principles for responsible sharing of clinical trial data, researchers who desire access to data from our clinical trials can make a request on "http://www.clinicaltrialstudytransparency.com".

\section{Authors' contributions}

$\mathrm{KY}, \mathrm{T}, \mathrm{HK}$ and MN were involved in the conception and design of the study. $\mathrm{RO}$ was responsible for the overall study project. MN and HK were responsible for statistical analysis. MN and KY drafted the manuscript and $\mathrm{KI}$ was responsible for medical and urological interpretation of the analyzed data of the study. All authors read and approved the final version of the manuscript.

\section{Competing interests}

Masahiko Nakayama, Hisanori Kobayashi, Tomihiro Takahara, Kazutake Yoshizawa, Ryo Oyama, and Keiichiro Imanaka are employees of Janssen Pharmaceutical K.K.

\section{Consent for publication}

\section{Not applicable.}

\section{Ethics approval and consent to participate}

An independent ethics committee or institutional review board approved the protocols, and the trials were conducted in accordance with the ethical principles in the Declaration of Helsinki and consistent with the Good Clinical Practices and applicable regulatory requirements. All patients provided written informed consent before entering the original trials.

\section{Author details}

'Medical Affairs Division, Janssen Pharmaceutical K.K., 5-2, Nishi-kanda, 3-Chome, Chiyoda-ku, Tokyo 101-0065, Japan. ${ }^{2}$ Research and Development Division, Janssen Pharmaceutical K.K., 5-2, Nishi-kanda, 3-Chome, Chiyoda-ku, Tokyo 101-0065, Japan.

Received: 28 January 2016 Accepted: 3 June 2016

Published online: 08 June 2016

\section{References}

1. Fitzmaurice C, Dicker D, Pain A, Hamavid H, Moradi-Lakeh M, Maclntyre MF, Allen C, Hansen G, Woodbrook R, Wolfe C, et al. The global burden of cancer 2013. JAMA Oncol. 2015;1(4):505-27.

2. Huggins $\mathrm{C}$, Masina MH, Eichelberger $\mathrm{L}$, Wharton JD. Quantitative studies of prostatic secretion : I. Characteristics of the normal secretion; the influence of thyroid, suprarenal, and tstis extirpation and androgen substitution on the prostatic output. J Exp Med. 1939;70(6):543-56.

3. Barrie SE, Potter GA, Goddard PM, Haynes BP, Dowsett M, Jarman M. Pharmacology of novel steroidal inhibitors of cytochrome P450(17) alpha (17 alpha-hydroxylase/C17-20 lyase). J Steroid Biochem Mol Biol. 1994; 50(5-6):267-73.

4. de Bono JS, Logothetis CJ, Molina A, Fizazi K, North S, Chu L, Chi KN, Jones RJ, Goodman OB, Jr., Saad F, et al. Abiraterone and increased survival in metastatic prostate cancer. N Engl J Med. 2011;364(21):1995-2005.
5. Ryan CJ, Smith MR, de Bono JS, Molina A, Logothetis CJ, de Souza P, Fizazi $\mathrm{K}$, Mainwaring P, Piulats JM, Ng S, et al. Abiraterone in metastatic prostate cancer without previous chemotherapy. N Engl J Med. 2013;368(2):138-48.

6. Satoh T, Uemura H, Tanabe K, Nishiyama T, Terai A, Yokomizo A, Nakatani T, Imanaka K, Ozono S, Akaza H. A phase 2 study of abiraterone acetate in Japanese men with metastatic castration-resistant prostate cancer who had received docetaxel-based chemotherapy. Jpn J Clin Oncol. 2014;44(12):1206-15.

7. Matsubara N, Uemura H, Satoh T, Suzuki H, Nishiyama T, Uemura H, Hashine K, Imanaka K, Ozono S, Akaza H. A phase 2 trial of abiraterone acetate in Japanese men with metastatic castration-resistant prostate cancer and without prior chemotherapy (JPN-201 study). Jpn J Clin Oncol. 2014;44(12):1216-26.

8. Scher HI, Halabi S, Tannock I, Morris M, Sternberg CN, Carducci MA, Eisenberger MA, Higano C, Bubley GJ, Dreicer R, et al. Design and end points of clinical trials for patients with progressive prostate cancer and castrate levels of testosterone: recommendations of the Prostate Cancer Clinical Trials Working Group. J Clin Oncol. 2008;26(7):1148-59.

9. Armstrong AJ, Eisenberger MA, Halabi S, Oudard S, Nanus DM, Petrylak DP, Sartor $\mathrm{AO}$, Scher $\mathrm{HI}$. Biomarkers in the management and treatment of men with metastatic castration-resistant prostate cancer. Eur Urol. 2012;61(3):549-59.

10. Collette L, Burzykowski T, Carroll KJ, Newling D, Morris T, Schroder FH. Is prostate-specific antigen a valid surrogate end point for survival in hormonally treated patients with metastatic prostate cancer? Joint research of the European Organisation for Research and Treatment of Cancer, the Limburgs Universitair Centrum, and AstraZeneca Pharmaceuticals. J Clin Oncol. 2005;23(25):6139-48.

11. Halabi S, Armstrong AJ, Sartor O, de Bono J, Kaplan E, Lin CY, Solomon NC, Small EJ. Prostate-specific antigen changes as surrogate for overall survival in men with metastatic castration-resistant prostate cancer treated with second-line chemotherapy. J Clin Oncol. 2013;31(31):3944-50.

12. Caffo O, Veccia A, Maines F, Bonetta A, Spizzo G, Galligioni E. Potential value of rapid prostate-specific antigen decline in identifying primary resistance to abiraterone acetate and enzalutamide. Future Oncol. 2014;10(6):985-93.

13. Xu XS, Ryan CJ, Stuyckens K, Smith MR, Saad F, Griffin TW, Park YC, Yu MK, Vermeulen A, Poggesi I, et al. Correlation between prostate-specific antigen kinetics and overall survival in abiraterone acetate-treated castration-resistant prostate cancer patients. Clin Cancer Res. 2015; 21(14):3170-7.

14. Guccione JR, Ledet EM, Stolten MD, Steinberger AE, Chow LD, Cotogno P, Lewis BE, Sartor AO. Early assessment of PSA response in CRPC patients treated with enzalutamide (Enza) or abiraterone (Abi). ASCO Meeting Abstracts. 2016;34(2_suppl):249.

15. Conteduca V, Crabb SJ, Scarpi E, Hanna C, Maines F, Joyce H, Fabbri P, Derosa L, Massari F, Lolli C, et al. Association between early PSA increase and clinical outcome in patients treated with enzalutamide for metastatic castration resistant prostate cancer. ASCO Meeting Abstracts. 2016;34(2_suppl):231.

16. Bubley GJ, Carducci M, Dahut W, Dawson N, Daliani D, Eisenberger M, Figg WD, Freidlin B, Halabi S, Hudes $G$, et al. Eligibility and response guidelines for phase II clinical trials in androgen-independent prostate cancer: recommendations from the Prostate-Specific Antigen Working Group. J Clin Oncol. 1999;17(11):3461-7.

17. Hoffman-Censits J, Kelly WK. Enzalutamide: a novel antiandrogen for patients with castrate-resistant prostate cancer. Clin Cancer Res. 2013; 19(6):1335-9.

18. Olbert PJ, Hegele A, Kraeuter P, Heidenreich A, Hofmann R, Schrader AJ. Clinical significance of a prostate-specific antigen flare phenomenon in patients with hormone-refractory prostate cancer receiving docetaxel. Anticancer Drugs. 2006;17(8):993-6.

19. Burgio SL, Conteduca V, Rudnas B, Carrozza F, Campadelli E, Bianchi E, Fabbri P, Montanari M, Carretta E, Menna C, et al. PSA flare with abiraterone in patients with metastatic castration-resistant prostate cancer. Clin Genitourin Cancer. 2015;13(1):39-43.

20. Narmala SK, Boulmay BC. PSA flare after initiation of abiraterone acetate. J Community Support Oncol. 2014;12(5):191-2.

21. Nelius T, Klatte T, de Riese W, Filleur S. Impact of PSA flare-up in patients with hormone-refractory prostate cancer undergoing chemotherapy. Int Urol Nephrol. 2008;40(1):97-104.

22. Angelergues A, Maillet D, Flechon A, Ozguroglu M, Mercier F, Guillot A, Le Moulec S, Gravis G, Beuzeboc P, Massard C, et al. Prostate-specific antigen flare induced by cabazitaxel-based chemotherapy in patients with metastatic castration-resistant prostate cancer. Eur J Cancer. 2014;50(9):1602-9. 